\title{
VOLUME INDEX
}

Arnold, David, Representations of finite posets over the ring of integers modulo a prime power, 461.

Axtell, M., Cut structures in zero-divisor graphs of commutative rings, 143.

Baeth, N., see Axtell, M.

Balakrishnan, R., A Northcott type inequality for Buchsbaum-Rim coefficients, 493.

Casnati, Gianfranco, A structure theorem for 2-stretched Gorenstein algebras, 295.

Chabert, Jean-Luc, Polynomial overrings of $\operatorname{Int}(\mathbb{Z}), 1$.

Chen, Ri-Xiang, On two classes of regular sequences, 29.

Diaz, Steven P., Polynomial foldings and rank of tensors, 173.

Evans, E. Graham, Order ideals, annihilator ideals and pathological behavior, 43.

Finocchiaro, Carmelo Antonio, On a topological characterization of Prüfer v-multiplication domains among essential domains, 513.

Freitas, T.H., On formal local cohomology modules with respect to a pair of ideals, 337.

Gheorghita, Iulia, The cone of Betti tables over three non-collinear points in the plane, 537.

Gimenez, Philippe, On complete monomial ideals, 207.

Griffith, Phillip, see Evans, E. Graham.

Hasenauer, Richard Erwin, Normsets of almost Dedekind domains and atomicity, 61.

Hoa, Le Tuan, Castelnuovo-Mumford regularity of symbolic powers of two-dimensional square-free monomial ideals, 77.

Isaev, A.V., A criterion for isomorphism of Artinian Gorenstein algebras, 89.

Jayanthan, A.V., see Balakrishnan, R.

Johnson, Mark R., Reduced symmetric algebras and linear syzygies, 227.

Lella, Paolo, On the functoriality of marked families, 367.

Loper, K. Alan, Pseudo-convergent sequences and Prüfer domains of integer-valued polynomials, 411.

Lutoborski, Adam, see Diaz, Steven P.

Mader, Adolf, see Arnold, David.

Maeno, Toshiaki, Sperner property and finite-dimensional Gorenstein algebras associated to matroids, 549.

Mutzbauer, Otto, see Arnold, David.

Notari, Roberto, see Casnati, Gianfranco.

Numata, Yasuhide, see Maeno, Toshiaki.

Olteanu, Anda, The Buchberger resolution, 571.

Pérez, V.H. Jorge, see Freitas, T.H.

Peruginelli, Giulio, The ring of polynomials integral-valued over a finite set of integral elements, 113.

Peruginelli, Giulio, see Chabert, Jean-Luc.

Robbins, Hannah, Associated primes of local cohomology after adjoining indeterminates. Part 2: The general case, 589.

Roggero, Margherita, see Lella, Paolo.

Sabzrou, Hossein, Multigraded modules of nested type, 431.

Sam, Steven V, see Gheorghita, Iulia.

Sammartano, Alessio, s-Hankel hypermatrices and $2 \times 2$ determinantal ideals, 239 .

Simis, Aron, see Gimenez, Philippe.

Solak, Ebru, see Arnold, David.

Stickles, J., see Axtell, M. 
Tartarone, Francesca, see Finocchiaro, Carmelo Antonio.

Totushek, Jonathan, Homological dimensions with respect to a semidualizing complex, 275.

Tousi, Massoud, see Sabzrou, Hossein.

Trung, Tran Nam, see Hoa, Le Tuan.

Vasconcelos, Wolmer V., see Gimenez, Philippe.

Villarreal, Rafael H., see Gimenez, Philippe.

Welker, Volkmar, see Olteanu, Anda.

Werner, Nicholas J., see Loper, K. Alan. 